\title{
Design and Mathematical Modeling of Gearless SMC Flux Reversal Motor
}

\author{
Vladimir Prakht \\ Department of Electrical Engineering \\ and Electric Technology Systems \\ Ural Federal University \\ Yekaterinburg, Russia \\ emf2010@mail.ru \\ Dmitry Golovanov \\ Department of Electrical Engineering \\ University of Nottingham \\ Nottingham, United Kingdom \\ dmitry.golovanov@nottingham.ac.uk
}

\author{
Vladimir Dmitrievskii \\ Department of Electrical Engineering \\ and Electric Technology Systems \\ Ural Federal University \\ Yekaterinburg, Russia \\ vdmitrievsky@gmail.com \\ David Gerada ${ }^{1,2}$ \\ ${ }^{I}$ Department of Electrical Engineering \\ University of Nottingham \\ Nottingham, United Kingdom \\ ${ }^{2}$ School of Engineering \\ University of Nottingham Ningbo \\ Ningbo, China \\ d.gerada@nottingham.ac.uk
}

\author{
Vadim Kazakbaev \\ Department of Electrical Engineering \\ and Electric Technology Systems \\ Ural Federal University \\ Yekaterinburg, Russia \\ kazakbaiev@inbox.ru
}

\begin{abstract}
This paper considers the design of a three-phase gearless flux reversal machine (FRM) with permanent magnets (PM) mounted on the stator. Its stator magnetic core is made of a solid soft magnetic composite material (SMC) with a slot for laying the winding in the middle. 48 magnetic poles are located on the surface of each stator tooth, while the rotor has 24 teeth. This paper describes the mathematical model and the results of the performance calculation of the three-phase SMC FRM operating in motoring mode. Finally, comparison of the SMC FRM with the characteristics of the conventional surface mounted permanent magnet machine (SM-PMSM) made of laminated steel is performed. For fair comparison, both the SMC FRM and SM-PMSM have the same outer stator diameter and approximately the same volume.
\end{abstract}

Keywords-gearless motor, machine modeling and general design, permanent magnet machines, soft magnetic composite materials, special machines

\section{INTRODUCTION}

Flux Reversal Machine (FRM) is a machine with PMs mounted on the stator inner surface where the flux linkage reverses its direction due to the alternating magnetic poles, creating useful magnetomotive force (MMF) while the rotor rotates.

Traditional constructions of single-phase Flux Reversal motors were made of laminated steel [1], [2], [3]. Papers [4] and [5] describe single-phase 8-pole FRM, in which magnetic cores are made of soft magnetic composite (SMC) material. This design also corresponds to the patent [6]. Paper [7] describes a 48-pole SMC FRM, which was designed according to the same patent.

Unlike the FRM made of laminated steel [1], [2], [3], the SMC FRM winding has no end parts. Thus the winding is entirely used for inducing MMF [7]. This contributes to the improvement of both the power-to-weight ratio as well as the efficiency of the machine. Besides, compared to traditional manufacturing techniques of the stator and rotor cores by means of punching electrical steel, use of SMC helps to reduce material wastage [7].

Fig. 1 schematically shows the design of the considered SMC FRM. The rotor and the stator both have SMC cores. The stator core ' 1 ' is a solid hollow cylinder with one slot

The research is done on theme no. № 8.9549.2017/8.9. within the frame of the government task of the Ministry of Education and Science of the Russian Federation in R\&D. containing the winding ' 3 '. Two sets of PMs are fixed on the stator teeth. 48 magnetic poles are fixed on each of the stator tooth surface so that the closest magnets located on different teeth have the opposite magnetization directions. The rotor core ' 2 ' has $r=24$ teeth. In Fig. 1, the number of poles is reduced for visual clarity. The fundamental frequency of the SMC FRM is $f=r \cdot n / 60[\mathrm{~Hz}]$, where $\mathrm{n}$ is the rotor speed $[\mathrm{rpm}]$.

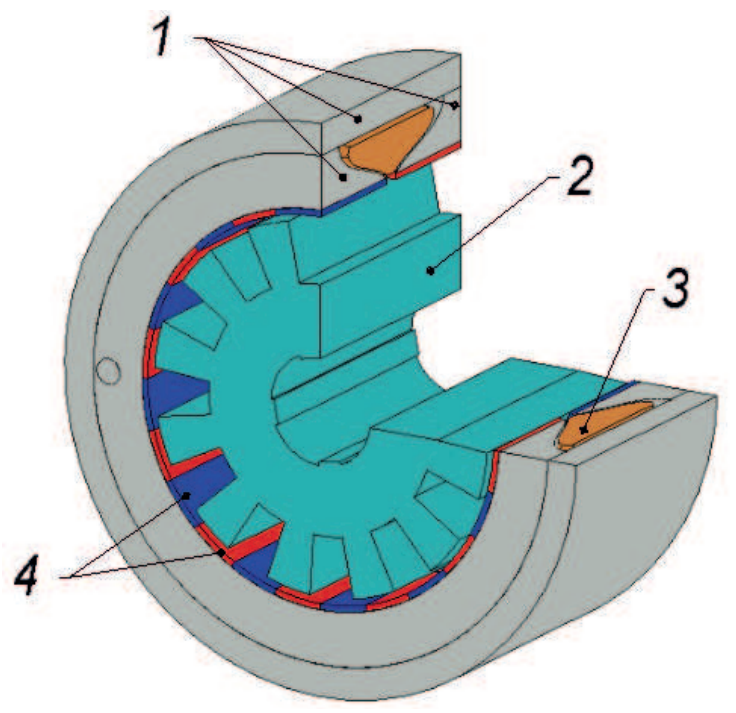

(a)

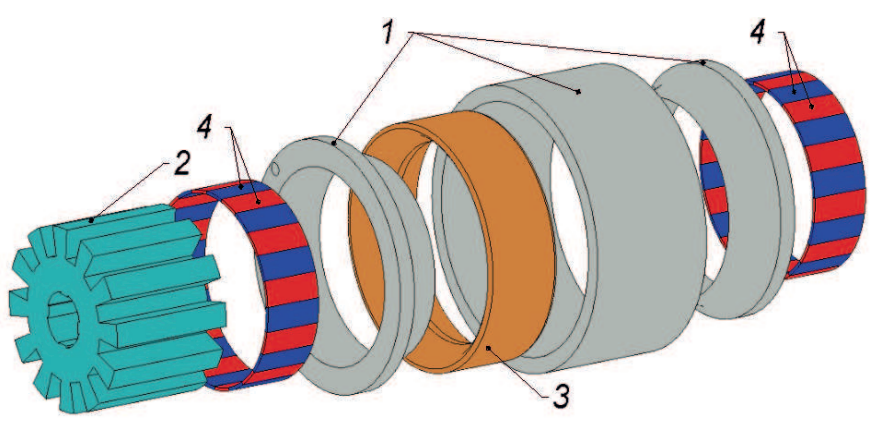

(b)

Fig. 1. Multipole SMC FRM design according [6].

Single-phase motors have a small starting torque and large torque ripple. Therefore, such machines have a limited 
range of applications, such as for example in circular saws, vacuum cleaners etc. where a large starting torque is not required. Three-phase motors have much lower torque ripple and can be used in a broader range of applications.

This paper considers the possibility of developing a three-phase, low-speed gearless motor based on the SMC FRM design described in [7]. This three-phase motor consists of three single-phase machines that are mounted on a single shaft, shifted relative to each other by 120 electrical degrees (see Fig. 2), i.e. 5 geometric degrees for the 48-pole motor, and are separated with an air span.

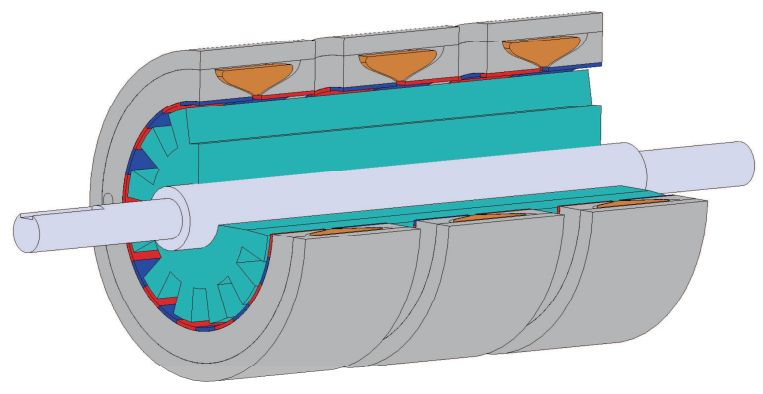

Fig. 2. The scheme of the 3-phase SMC FRM.

The operating principle of the SMC FRM in motoring mode is described below. When rotor teeth are between the stator poles, the armature current magnetically strengthens some of these poles and weakens others. The rotor rotates by attraction to the stronger poles. For the torque to have one and the same direction during the most of the time, the winding current should change its direction.

The 3-phase SMC FRM control is carried out using a conventional three-phase frequency converter with a common point. As a result, each FRM phase is controlled independently with respect to the others. The frequency converter generates rectangular voltage pulses that consistently take the following values: $0,320 \mathrm{~V}, 0,-320 \mathrm{~V}$.

This paper describes the mathematical model and the results of calculating performances of the three-phase SMC FRM.

The torque waveform of the 3-phase SMC FRM is studied on the basis of the torque waveform of a singlephase SMC FRM. The torque waveform of single-phase SMC FRM is plotted with more points than in [7] because in the 3-phase SMC FRM the long-wave harmonics of the torque waveform disappear and the short-wave harmonics with smaller amplitude play a major role.

This leads to increased computational efforts. Therefore the paper contains a short description related to issues of mathematical modeling using a scalar magnetic potential and exploiting the machine symmetry.

To evaluate the advantages of the gearless SMC FRM, a comparison of the calculated characteristics of the considered machine with the calculated characteristics of conventional SM-PMSM, is given. For fair comparison, both SMC FRM and SM-PMSM have the same outer stator diameter $(110 \mathrm{~mm})$ and approximately the same volume.

\section{Mathematical Modeling The Three-Phase FRM}

\section{A. Short Description of The SMC FRM Mathematical Model}

The electric circuit equation takes the form (1):

$$
U=\frac{d \Phi}{d t}+I \cdot r
$$

where $U$ is voltage, $\Phi$ denotes flux linkage, $I$ is the winding current, $r$ is winding resistance, and $t$ denotes time.

Neglecting the winding resistance, we obtain that the time dependence of flux linkage is determined by the time dependence of voltage as (2):

$$
\Phi=\int U d t
$$

The FRM operation is symmetric with respect to rotating the rotor by half a period and simultaneously changing the signs of the magnetization direction, the slot current and the magnetic field. In particular, $\Phi(t)=-\Phi(t+T / 2)$, where $T$ is a period. Hence, the integration constant in (2) can be chosen so that $\langle\Phi\rangle=0$.

The controlled rectifier forms a piecewise constant voltage for each machine phase, which sequentially takes the values of $0 \mathrm{~V}, 320 \mathrm{~V}, 0 \mathrm{~V},-320 \mathrm{~V}$, with the time intervals at $320 \mathrm{~V}$ and $-320 \mathrm{~V}$ being of equal duration. The time intervals at zero voltage are also of equal duration, thus the voltage can be considered antiperiodic.

According to (2) the flux linkage is a piecewise linear function the period of which comprises two parts of constant flux linkage corresponding to zero voltage and two parts of linearly changing flux linkage corresponding to nonzero voltages. The slope of the linear functions is the same except for the sign. The fluxes at constant intervals are also the same except for sign. Besides, the assumption of uniform rotor rotation determines the dependence of the time on the rotor position and, in the end determines the dependence of the flux linkage on the rotor position.

Hence, the boundary problems can be formulated as follows: for each angle and for corresponding flux linkage to this angle, the magnetostatic field is required to be calculated.

The scheme of the calculation area is shown in Fig. 3. There are two symmetries allowing for the reduction in the computational area: mirror symmetry and rotational symmetry.

1. Mirror symmetry. SMC FRM is symmetric in the plane perpendicular to the rotational axis and passing through the FRM's middle. Indeed, this operation transfers the air, the winding, the rotor and stator magnetic cores into themselves. The same happens with the magnets taking into account that the magnetization is a pseudo vector and the magnet being mirror-reflected changes its direction. This symmetry halves the calculation area. 
2. The other symmetry is the rotational one. Independent of the rotor position the SMC FRM with $n$ teeth on the rotor and $2 n$ magnetic poles at each of the stator teeth coincides with itself being rotated by an angle of $360^{\circ} / n$ as a whole. Hence only the $n^{\text {th }}$ part of FRM bounded by two radial planes can be considered. The periodic joining condition should be applied on these planes. For this purpose, the values of $u$ on the corresponding points of these radial planes should be equal to each other. This symmetry reduces the calculation area by $n$ times.

In light of the aforesaid, exploiting all symmetries the calculation area is reduced by $2 n$ times.

The same calculation area is created for all boundary problems corresponding to various rotor positions. The calculation area is divided into two areas: the stator area containing the stator together with half of the air gap and the rotor area containing the rotor and the other half of the air gap. The common boundary of these two areas is in the middle of the air gap. The rotor position is taken into account by the joining boundary condition on the common boundary of these areas.

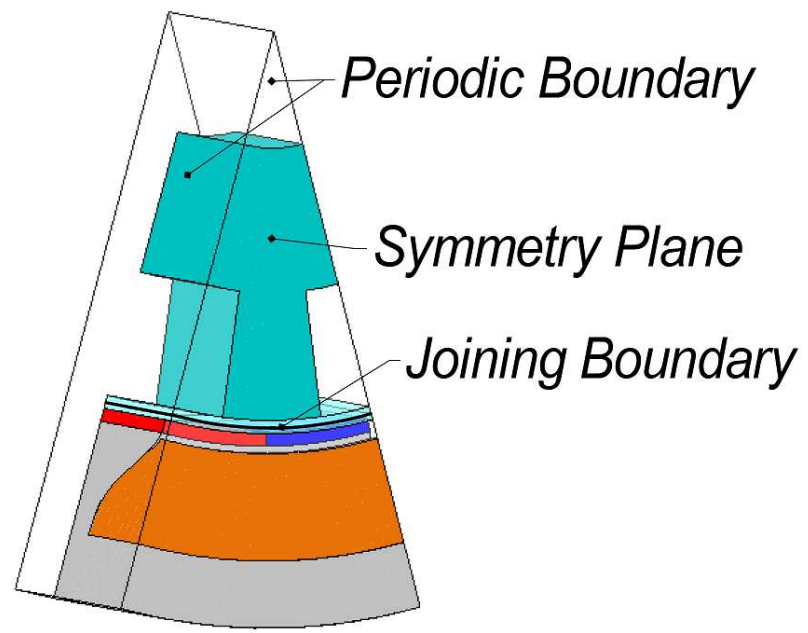

Fig. 3. The scheme of the calculation area of the SMC FRM.

SMCs consist of magnetic particles separated from each other with epoxy which prevents the flow of eddy current through the magnetic cores. Hence the core losses are hysteresis-dominated. The hysteresis losses depend on the flux density time-derivatives and not on the electric field in the cores. This makes it possible to use scalar magnetic potential having only one field component instead of vector magnetic potential having three which simplifies the resource-consumptive 3D calculations.

In the rotor area, since there are no currents, the magnetic field $\mathbf{H}$ is a potential field: $\mathbf{H}=-\nabla u$, where $u$ is a scalar potential.

In stator area the current density $\mathbf{J}$ is concentrated in the winding area. So, if the field $\mathbf{K}$ satisfying the equation $\nabla \times \mathbf{K}=\mathbf{J}$ is constructed, $\mathbf{H}=\mathbf{K}-\nabla u$. The joining condition on the common boundary of two subareas makes $u$ to be equal on both side of this boundary taking into account that the rotation sets a correspondence between points on one and another sides of the common boundary. This joining condition is considered in [7] in more detail. At the mirror boundary, $u=0$ since $u$ is a pseudoscalar.
The field $\mathbf{K}$ should not have tangential components at the mirror boundary due to it being a pseudo-vector and at the common boundary of the rotor and stator areas since there is no $\mathbf{K}$-field in the rotor boundary and tangential components of $\mathbf{K}$ would mean the current in the common boundary. The periodic condition should be applied at the periodic boundaries.

Such vector field $\mathbf{K}$ is constructed to be proportional to the winding current $I: \mathbf{K}=\boldsymbol{\Xi} I$, where $\boldsymbol{\Xi}$ is $\mathbf{K}$ - field for $I=1 A$.

The flux linkage is determined from the equation:

$$
\Phi=2 n \iiint \mathbf{\Xi} \mathbf{B} d V
$$

where $2 n$ is the multiplier taking into account the reduction of the calculation area.

Equation (3) should be considered as another equation in addition to the set of equations obtained from the magnetic field submodel discretization. The winding current corresponds to an additional degree of freedom.

\section{B. Postprocessing}

The torque is calculated and the losses are evaluated in postprocessing.

For the aim of convenience the subdomains of the halves of air gap belonging to both areas (I and II) are spread through entire model geometry in axial direction including not only the active zone but also the surrounding air and have cylindrical form.

The torque calculation is carried out by Maxwell's tension integration. This integration can be carried out on any surface in the air gap. Averaging the values gotten by integrating on the concentric cylinders in the air gap allows getting more accurate result, so we have integral on the air gap including its both halves (4):

$$
M=\iiint_{\substack{\text { gap } \\ \text { domain }}} \frac{x y\left(B_{y}^{2}-B_{x}^{2}\right)+\left(x^{2}-y^{2}\right) B_{x} B_{y}}{\delta_{\text {air }} \mu_{0} \sqrt{x^{2}+y^{2}}} d V
$$

The integration in (4) should be carried out in the air gap subdomain that is area including air gap parts belonging to both subareas.

Knowing current dependence on time, the winding losses are calculated according to Joule-Lenz law.

The core losses are expressed through the magnetic field total derivative. As far as rotor and stator are considered in their reference frame, the total derivative coincides with partial derivative which significantly simplifies the calculation.

The experimental data of the SMC losses volume density are available for sinusoidal flux density and can be approximated as following (5):

$$
p=\alpha f^{k} B^{2}
$$


where $\alpha$ and $k$ are parameters, $f$ is frequency and $B$ is flux density amplitude.

The expression (6) is used for core losses estimation in this paper:

$$
p=k<\left(\frac{\partial \mathbf{B}}{\partial t}\right)^{2}>
$$

where $<>$ is the averaging sign, factor $k$ depending on supply frequency $f$ is chosen so that (5) and (6) coincide for the basic harmonic (7):

$$
k=\frac{\alpha f^{n-2}}{2 \pi^{2}} .
$$

\section{THE SMC FRM MODELING RESULTS}

The rated rotational speed of the considered SMC FRM is $650 \mathrm{rpm}$, the rated mechanical power is $600 \mathrm{~W}$, while the fundamental frequency is $260 \mathrm{~Hz}$.

Fig. 4 shows the torque harmonic spectrum of one phase of the machine. The period of the torque (half the period of the electrical cycle, 180 electrical degrees, i.e. $7.5^{\circ}$ for the 48-pole motor) contains one period of the first torque harmonic, two periods of the second harmonic, etc. The average value of the torque is $2.94 \mathrm{~N} \cdot \mathrm{m}$. Fig. 4 shows that the torque harmonic spectrum is dominated by the $1^{\text {st }}$ and $2^{\text {nd }}$ harmonics. Therefore, only a small number of points are required to estimate the torque harmonic spectrum correctly. In fact paper [7] contains a single-phase machine torque-plot with 10 points per period.

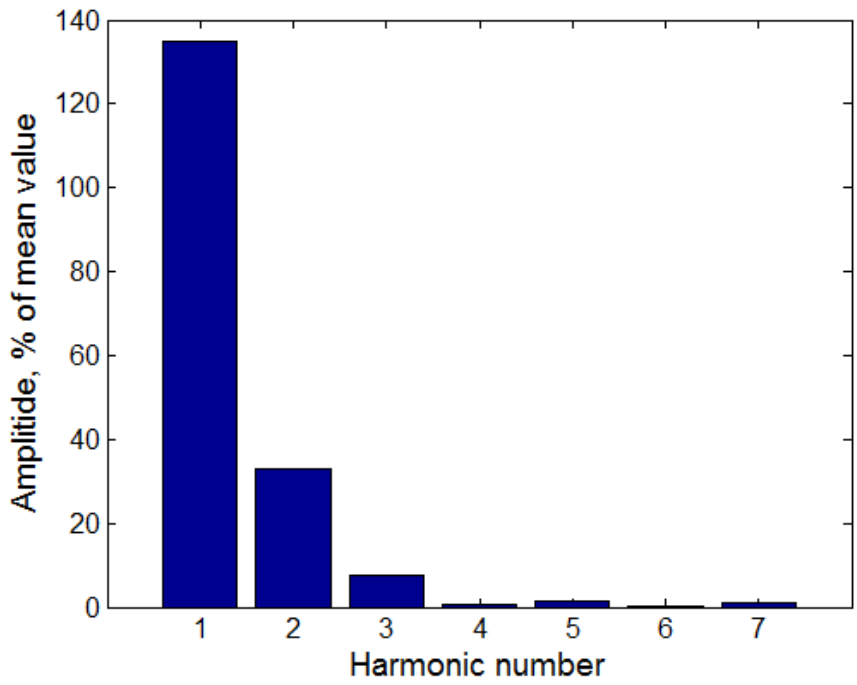

Fig. 4. Torque waveform harmonics of the single-phase SMC FRM.

To obtain the dependence of the torque with the rotational angle of the three-phase SMC FRM composed of three single-phase motors, it is necessary to add up the torque components for each of the constituent single-phase motors. These torque waveforms coincide with each other, except that they are shifted relative to each other by a third of the electric period.
In the three-phase motor composed of three single-phase machines, non-triplen harmonics are eliminated, in particular the $1^{\text {st }}$ and $2^{\text {nd }}$ harmonics. Therefore, torque pulsations are due to harmonics with a smaller period, and the number of calculated points should thus be increased.

Fig. 5 shows the graph of the torque dependence on the rotor position, which was developed on 42 points for the torque period, i.e. on $\left[0,7.5^{\circ}\right]$.

The number of points is a multiple of three. Therefore, the shift by 120 electrical degrees corresponds to the shift by an integer number of points $42 / 3=14$, which allows avoiding interpolation in case of such shift.

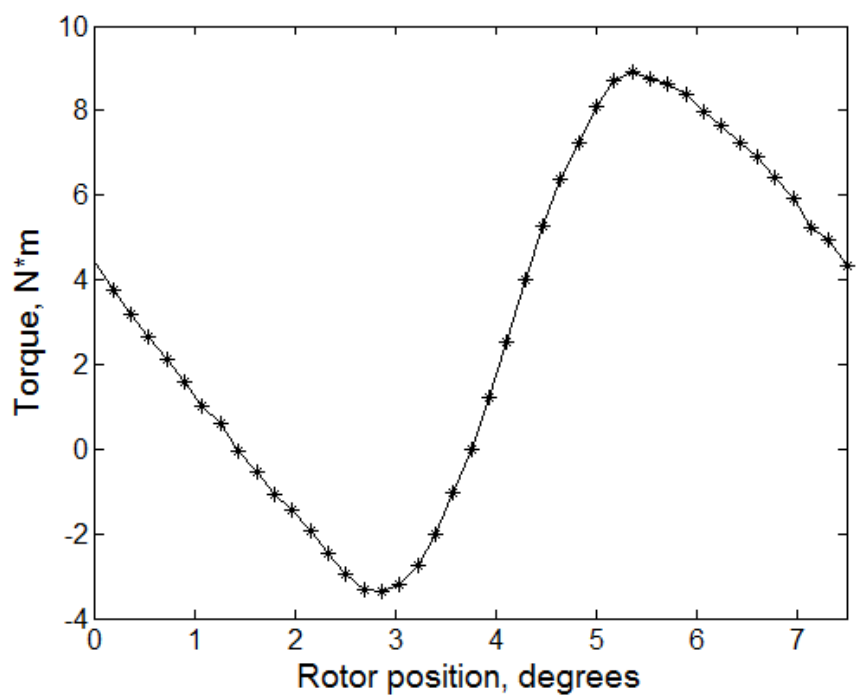

Fig. 5. Torque waveform containing 42 calculated points per period of the single-phase SMC FRM.

Fig. 6 shows the torque of each of the three motors placed on the common shaft.

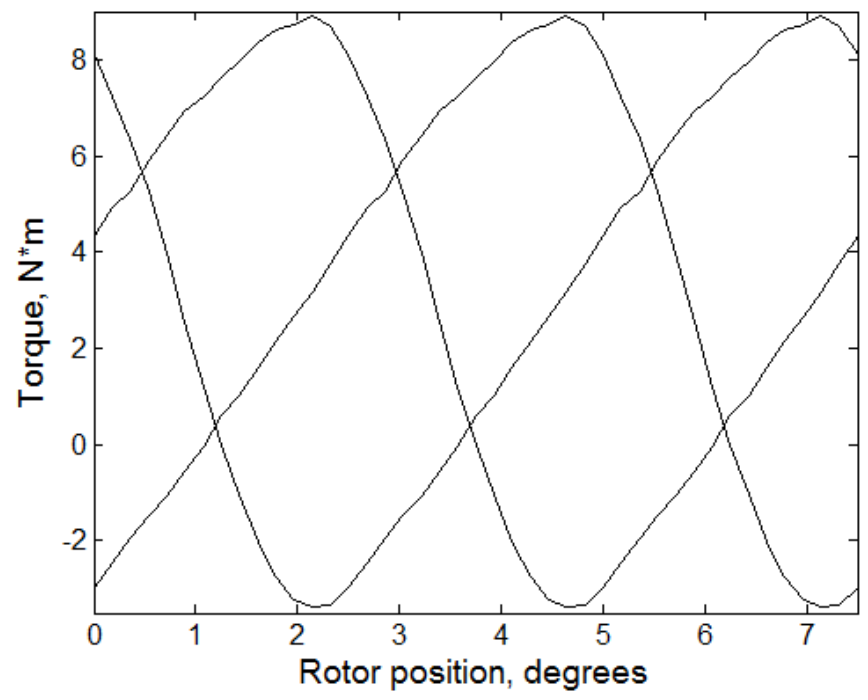

Fig. 6. Torque waveforms of individual single-phase machines.

As a result of summing up the torques from the three separate motors, the net torque of the three-phase motor is obtained (see Fig. 7). The average value of the torque is 8.81 $\mathrm{N} \cdot \mathrm{m}$. The period of the torque waveform is 60 electrical degrees or $2.5^{\circ}$, with the torque ripple at rated mode being $20 \%$. 


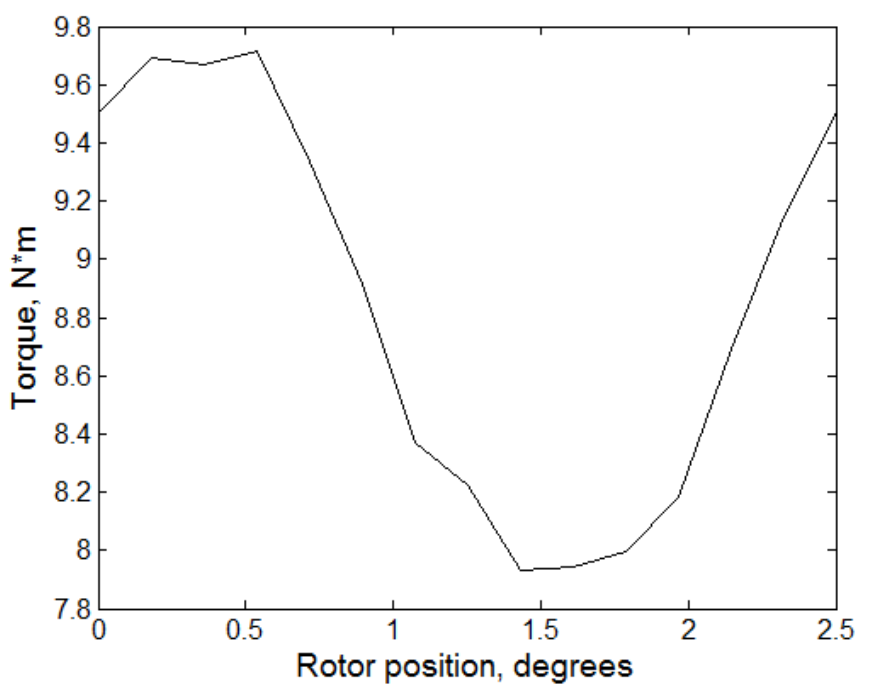

Fig. 7. Torque waveform at the rated load of the three-phase SMC FRM.

\section{COMPARISON BETWEEN THE SMC FRM AND AN SM- PMSM GEARLESS MOTOR}

In this section the performance characteristics of the gearless SMC FRM are compared to the ones of a conventional SM-PMSM machine for the same speed and power levels.

Multi-pole synchronous machines with magnets on the rotor are commonly used in gearless drives and wind turbine generators. However, in the case of fractional-horsepower machines, making such synchronous machines of small outer diameter is not always easy for high pole numbers. For example, the gearless synchronous motor of similar power and speed (500 rpm, $390 \mathrm{~W}, 130 \mathrm{~mm}$ outer diameter, model 145 STK Motors) with distributed winding [8] has 12 poles on its rotor. Also, another commercially available motor [10] with distributed winding the diameter of which is 124 $\mathrm{mm}$ and with the number of slots per pole per phase ' $q$ ' being 1 has only 8 poles. In such cases further increasing the number of the poles is difficult because it leads to an increase of the number of stator slots, thus decreasing the copper fill factor and, as a result, decreasing the efficiency.

For machines with a small outer diameter, multi-pole synchronous machines with concentrated windings can have a higher pole number than those with a distributed winding. For example, the synchronous machines in [7] (3000 rpm, $1100 \mathrm{~W}, 110 \mathrm{~mm}$ of outer diameter) has 16 poles on the rotor. However, the synchronous machines with concentrated winding can exhibit higher acoustic noise, higher torque ripple, and non-sinusoidal magneto-motive force distribution in the air gap which can worsen the motors' specific power and torque.

Therefore in this paper, the 8-pole motor with magnets on the rotor and with a distributed winding $q=1$ was designed to be compared to the SMC FRM.

A Genetic Algorithm (GA) which is embedded in the commercial optimization software mode Frontier ${ }^{\mathrm{TM}}$ coupled with a Matlab script is used to determine the optimum design of SM-PMSM [9]. The Matlab script estimates electromagnetic parameters of the machine as well as the mass of the passive and active parts. The machine outer diameter and core length were taken equal to $110 \mathrm{~mm}$ and
$70 \mathrm{~mm}$ respectively. Slot width, slot height, magnet height and stator bore diameter were considered as input variables for optimization. The results of optimization were then validated in commercial FEA software. The SM-PMSM machine design is shown in Fig. 8.

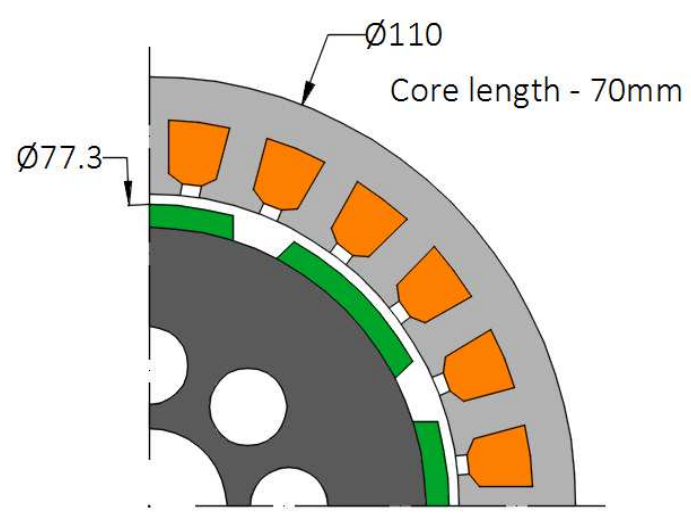

Fig. 8. 8-pole, 24-slot SM-PMSM machine.

Table 1 shows key features of the compared machines.

TABLE I. THE SPECIFICATIONS OF $600 \mathrm{~W} 650$ RPM GEARLESS MOTORS

\begin{tabular}{|c|c|c|}
\hline Parameter & SMC FRM & $\begin{array}{c}\text { Motor with } \\
\text { Magnets on } \\
\text { The Rotor }\end{array}$ \\
\hline Outer diameter of the stator core, $\mathrm{mm}$ & 110 & 110 \\
\hline Length of the cores, $\mathrm{mm}$ & 84 & 70 \\
\hline Volume of the active part, liters & 0.8 & 1.1 \\
\hline $\begin{array}{l}\text { Protrusion of the wining end parts, } \\
\mathrm{mm}\end{array}$ & - & 22.5 \\
\hline Active parts length, $\mathrm{mm}$ & 84 & 115 \\
\hline Magnetic core material & $\begin{array}{l}\text { Somaloy } \\
10003 \mathrm{P}\end{array}$ & M350-50A \\
\hline Magnet grade & N35UH & N35UH \\
\hline Remanent flux density, $\mathrm{T}$ & 1.2 & 1.2 \\
\hline Current density, $\mathrm{A} / \mathrm{mm}^{2}$ & 4.7 & 11.7 \\
\hline Rotational speed, rpm & 650 & 650 \\
\hline Rated mechanical power, W & 600 & 600 \\
\hline Eff $f_{1}$ (torque $100 \%$, speed $\left.100 \%\right)$ & 85.6 & 75.8 \\
\hline $\mathrm{Eff}_{2}($ torque $50 \%$, speed $75 \%)$ & 81.6 & 80.8 \\
\hline $\mathrm{Eff}_{3}$ (torque $25 \%$, speed $50 \%$ ) & 71.5 & 81.6 \\
\hline Basic frequency, $\mathrm{Hz}$ & 260 & 43 \\
\hline Torque ripple, $\%$ & 20 & 14.8 \\
\hline Rotor diameter, $\mathrm{mm}$ & 76 & 77.3 \\
\hline Rotor core mass, $\mathrm{g}$ & 1640 & 1640 \\
\hline Mass of the permanent magnets, $g$ & 130 & 270 \\
\hline Stator core mass, $\mathrm{g}$ & 2310 & 1610 \\
\hline Copper mass, $g$ & 360 & 530 \\
\hline Total active material mass, $\mathrm{g}$ & 4440 & 4050 \\
\hline
\end{tabular}


In Table 1 Eff $f_{l}$ is the efficiency at the rated speed and the rated torque, Eff 2 is the efficiency at the speed equal to $75 \%$ of the rated speed and the torque equal to $50 \%$ of the rated torque, $\mathrm{Eff}_{3}$ is the efficiency at the speed equal to $50 \%$ of the rated speed and the torque equal to $25 \%$ of the rated torque.

The number of poles formed by the magnets on the surface of the SMC FRM stator core can be increased easily without losing winding MMF, which leads to the considerable increase in torque, whereas increasing the number of poles in the motor made of the laminated steel results in decreasing the coil size and consequently MMF and copper fill factor [3]. Therefore SMC FRM has 48 poles whereas manufacturing a small-size, distributed-wound SMPMSM with number of poles much greater than 8 is more challenging.

From the results of the calculations, the mass of the permanent magnets of the SMC FRM is reduced by $140 \mathrm{~g}$ (more than twice), thus potentially contributing to costsavings, while the total mass of active material is increased by $390 \mathrm{~g}(10 \%)$. The volume of the active parts is reduced by $38 \%$.

Due to absence of winding overhang in SMC FRM, the required mass of copper is reduced by 1.47 times. The efficiency of the SMC FRM at the rated mode is $9.8 \%$ higher than the efficiency of the motor with magnets on the stator. It should be also mentioned that the current density of the reference SM-PMSM machine is almost $11.7 \mathrm{~A} / \mathrm{mm}^{2}$ against $4.7 \mathrm{~A} / \mathrm{mm}^{2}$ for SMC FRM. Since the dimension of active part was constrained this may cause some difficulties with cooling of the SM-PMSM.

It should be noted that designing the SM-PMSM was done with the help of an optimization procedure while no optimization procedures were used in designing SMC FRM. Therefore, further specific improvements of the SMC FRM performance are possible.

\section{CONCLUSION}

Gearless three-phase SMC FRM and SM-PMSM made of laminated steel were designed for a fractional-horsepower fan load application and compared in this paper. Both motors have the same rated power and speed $(600 \mathrm{~W}$ and $650 \mathrm{rpm})$.

Since the considered three-phase SMC FRM consists of three independent single-phase SMC FRM mounted on the same shaft, the mathematical model of single-phase SMC FRM is used for the design of this motor. However, calculation of three-phase SMC FRM torque waveform and torque ripple requires more significant computational effort because higher torque harmonics should be considered. Therefore, methods of decreasing computational time such as using scalar magnetic potential and motor symmetry were described in the paper.

Taking the fractional-horsepower case study investigated in this paper, compared to the SM-PMSM, the use of SMC FRM machine leads magnet cost-saving, while achieving better efficiencies at rated operation.

\section{REFERENCES}

[1] R. Deodhar, S. Andersson , I. Boldea, T. Miller, "The flux-reversal machine: a new brushless doubly-salient permanent-magnet machine", in IEEE Transactions On Industry Application, vol. 33, no. 4, pp. 925-934, July/August, 1997.

[2] Ki Bong Jang, Sung Hong Won, Tae Heoung Kim, and Ju Lee, "Starting and high-speed driving of single-phase flux-reversal motor for vacuum cleaner", IEEE Transactions On Magnetics, vol. 41, no. 10, October 2005

[3] V. Dmitrievskii, V. Prakht, A. Pozdeev, V. Klimarev, A. Mikhalitsyn, "Angular Grinder with New Flux Reversal Motor", in Proceedings of the 18th International Conference on Electrical Machines and Systems (ICEMS), pp. 1366-1371, Pattaya, Thailand, 2015.

[4] V. Dmitrievskii, V. Prakht, A. Mikhalitsyn, "A new single-phase flux reversal motor with the cores made of soft magnetic composite materials", in Proceedings of Electrical Machines and Systems (ICEMS), 2015 18th International Conference, pp. 936-939, Pattaya, Thailand, 25-28 Oct. 2015.

[5] V. Dmitrievskii, V. Prakht, A. Mikhalitsyn, S. Sarapulov, "Mathematical modeling and designing the flux reversal motor made of soft magnetic material", in Proceedings of 8th IET International Conference on Power Electronics, Machines and Drives, PEMD 2016, pp. 1-6, Glasgow, United Kingdom, 19-21 April 2016.

[6] Prakht V. A., Dmitrievskii V. A. "Brushless electric machine," Patent № RU 2526846.

[7] V. Dmitrievskii, V. Prakht, S. Sarapulov, D. Askerov, "A Multipole Single-Phase SMC Flux Reversal Motor For Fans", in Proceedings of the XXII International Conference on Electrical Machines (ICEM), pp. 53-59, Lausanne, Switzerland, 4-7 September 2016.

[8] Frameless permanent magnets brushless torque motors for direct drive Alxion STK, Alxion Automatique \& Productique, catalogue, Edition November 2017 - Revision 5.01.

[9] Dmitry Golovanov, Luca Papini, David Gerada, Zeyuan Xu, Chris Gerada, "Multi-domain Optimization of High Power-Density PM Electrical Machines for System Architecture Selection", IEEE Transaction on industrial electronics, 2017.

[10] SPRiPM Beyond Super Premium Efficiency, Hyosung corporation, Power \& Industrial Systems Performance Group, catalogue, March 2014. 